\title{
An ultrasonic methodology to non-destructively estimate the grain orientation in an anisotropic weld
}

\author{
Håkan Wirdelius ${ }^{\mathrm{a}}$ and Qingwei Liu \\ SCeNDT/MMT, Chalmers University of Technology, SE-412 96 Göteborg, Sweden
}

\begin{abstract}
The initial step towards a non-destructive technique that estimates grain orientation in an anisotropic weld is presented in this paper. The purpose is to aid future forward simulations of ultrasonic NDT of this kind of weld to achieve a better result. A forward model that consists of a weld model, a transmitter model, a receiver model and a $2 \mathrm{D}$ ray tracing algorithm is introduced. An inversion based on a multi-objective genetic algorithm is also presented. Experiments are conducted for both P and SV waves in order to collect enough data used in the inversion. Calculation is conducted to fulfil the estimation with both the synthetic data and the experimental data. Concluding remarks are presented at the end of the paper.
\end{abstract}

\section{Introduction}

Ultrasonic non-destructive testing (NDT) is widely applied in e.g. nuclear and aviation industry to assess integrity of vital components. Its capability to detect, characterize and size defects is also a tool to assess the quality of manufactured products. In the area of material science, the determination of elastic properties for a specific composite material is often essential. In many cases, an ultrasonic NDT technique is applied, and a direct measurement of ultrasound velocity is performed. Wang [1] presented a method to recover elastic constants from group velocity measurements in two dimensions while Aristégui et al. [2] presented a method that makes use of phase velocity measurement for optimal identification of material symmetry and independent elastic constants in a homogeneous anisotropic solid. Degtyar et al. [3] introduced an inversion procedure based on a nonlinear least-squares method to determine elastic constants from group or phase velocity data in orthotropic and transversely isotropic materials.

In many applications within the nuclear power industry, defects in or in the region of austenitic welds are assessed by using ultrasonic NDT. Since these welds tend to exhibit strong anisotropy, problems with the interpretation of received signal have been reported. When applying ultrasonic NDT to test their integrity, the propagation of ultrasound is significantly different from that in an isotropic medium. It is indicated by the fact that wave velocities become directionally dependent; group and phase velocities are no longer necessarily parallel or equal in magnitude [4].

\footnotetext{
${ }^{\text {a } C o r r e s p o n d i n g ~ a u t h o r: ~ h a k a n . w i r d e l i u s @ c h a l m e r s . s e ~}$
}

This is an Open Access article distributed under the terms of the Creative Commons Attribution License 4.0, which permits unrestricted use, distribution, and reproduction in any medium, provided the original work is properly cited. 


\section{MATEC Web of Conferences}



Figure 1. Subregions having a grain orientation to be determined in the inversion.

The assumption of the weld being two dimensional and transversely isotropic is often used [5, 6] and has recently also been experimentally validated [7]. Furthermore, the grain orientation is believed to be an essential factor that affects the propagation of ultrasound. In most simulation cases, a simple weld model is created by studying the macrograph of an austenitic weld. Different algorithms are then utilized to simulate the propagation of ultrasound through weld models.

In this paper, a procedure is described that estimates the grain orientation in an anisotropic austenitic weld based on ultrasonic information. In the study, the weld is divided into around eighty subregions. Each subregion is supposed to be homogeneous and transversely isotropic with its own crystal orientation. Combined with a ray tracing algorithm and experimental data, optimization calculations are utilized to further estimate these orientations.

\section{Forward model}

To establish a forward model, each element of the system is abstracted to a mathematical description. Then the output of the whole system is obtained on condition that a proper input is supplied to the model. Obviously, simulation results depend heavily on the accuracy of the model, as well as how closely the model approaches reality. In this paper, the forward model is implemented by a $2 \mathrm{D}$ ray tracing algorithm running on a weld model. The forward model then consists of a model of the weld, a model of the transmitter, a model of the receiver and a ray tracing model.

The weld model is created by analyzing the macrograph of a specific austenitic weld and the determination of the subregions and the crystal orientations is described in a previous paper [8]. The weld model created is shown in Fig. 1 and it consists of the base materials and eighty smaller weld subregions.

\section{References}

[1] L. Wang, J. Phys.: Condens. Matter 7, 3863-3880 (1995)

[2] C. Aristégui, S. Baste, J. Acoust. Soc. Am. 101(2), 813-833 (1997)

[3] A.D. Degtyar, S.I. Rokhlin, J. Acoust. Soc. Am. 102(6), 3458-3466 (1997)

[4] J.A. Ogilvy, NDT International 18(2), 67-77 (1985)

[5] B. Köhler, W. Müller, M. Spies, V. Schmitz, A. Zimmer, K.J. Langenberg, U. Metzko, AIP Conf. Proc. 820, 57-64 (2006) 
[6] K. Nakahata, S, Hirose, F. Schubert. B. Köhler, Journal of Solid Mechanics and Materials Engineering 3(12), 1256-1262 (2009)

[7] B. Chassignole, R. Guerjouma, M.A. Ploix, T. Fouquet, NDT \& E International 43(4), 273-282 (2011)

[8] Q. Liu, H. Wirdelius, NDT \& E International 40(3), 229-238 (2007) 